\title{
Some Considerations on the Nonlinear Stability of Stationary Planar Euler Flows ${ }^{\star}$
}

\author{
C. Marchioro ${ }^{1}$ and M. Pulvirenti ${ }^{2}$ \\ 1 Dipartimento di Matematica dell'Università di Trento, \\ I-38050 Povo (Trento), Italy \\ 2 Dipartimento di Matematica dell'Università di Roma \\ "La Sapienza," Piazzale Aldo Moro 2, I-00185 Roma, Italy
}

\begin{abstract}
We give sufficient conditions for the nonlinear stability of possibly nonsmooth stationary solutions of the two-dimensional Euler equation in symmetric bounded domains. We use, as Lyapunov functions, first integrals due to the symmetry of the problem. Moreover, we investigate the stability of smooth solutions under perturbations of the boundary. The last result is based on a generalization of the well known Arnold approach.
\end{abstract}

\section{1.}

Some years ago Arnold [1] proposed an approach to investigate the nonlinear stability of stationary Euler flows. According to the theory of finite dimensional Hamiltonian systems, the basic idea was to look for conditions ensuring the vanishing of the first variation of the energy functional and the positivity of its second variation. We briefly review the argument. For a more complete analysis we address the reader to [2], where other infinite-dimensional situations are also discussed.

Consider an incompressible ideal fluid contained in a domain $D$ bounded by two smooth curves $\mathscr{C}_{1}$ and $\mathscr{C}_{0}$, which are the internal and external boundary respectively. Then the following functional

$$
\hat{H}=\frac{1}{2} \int_{D} u^{2} d x d y+\int_{D} \Phi(\omega) d x d y+\sum_{i=0}^{1} a_{i} \oint_{\mathscr{C}_{i}} u \cdot d \ell
$$

( $u$ is the velocity field, $\omega=\operatorname{curl} u=\partial_{x} u^{(2)}-\partial_{y} u^{(1)}, \Phi$ a real valued function, $a_{i}$ real numbers) is a constant of motion, each of the three terms appearing in the righthand side of (1.1) being first integrals.

The condition $\delta \hat{H}(\bar{u})=0$, for $\bar{u}$ stationary solution, yields (see [2] for details)

$$
\begin{aligned}
& a_{i}=-\Phi^{\prime}\left(\left.\bar{\omega}\right|_{\mathscr{C}_{i}}\right) \quad(\bar{\omega}=\operatorname{cur} 1 \bar{u}), \\
& \bar{u}=\nabla^{\perp} \Phi^{\prime}(\bar{\omega}) \quad \nabla^{\perp}=\left(\partial_{y},-\partial_{x}\right) .
\end{aligned}
$$

* Research partially supported by Italian CNR and Ministero della Pubblica Istruzione 
(We notice that $\bar{\omega}$ is constant on $\mathscr{C}_{i}$ because $\bar{u}$ is stationary and hence $\bar{u} \perp \nabla \bar{\omega}$.)

Equation (1.3) can be solved and we obtain, for the solution $\Phi$,

$$
\Phi^{\prime \prime}(\bar{\omega})=\frac{\nabla \bar{\Psi}}{\nabla \bar{\omega}}=\frac{\bar{u}}{\nabla^{\perp} \bar{\omega}},
$$

where $\bar{\Psi}$ is the stream function (i.e., $\bar{u}=\nabla^{\perp} \bar{\Psi}$ ) and the above ratios are well defined by virtue of the collinearity of $\bar{u}$ and $\nabla^{\perp} \bar{\omega}$ for stationary solutions.

The second variation is

$$
\left(\delta^{2} \hat{H}\right)(\bar{u})=\int_{D}(\delta \bar{u})^{2} d x d y+\int_{D} \Phi^{\prime \prime}(\bar{\omega})(\delta \bar{\omega})^{2} d x d y,
$$

which is definite positive if $\Phi^{\prime \prime}>0$. In this case we have a natural norm that is $\int_{D}\left(u^{2}+\omega^{2}\right)$, by means of which we investigate the stability problem.

Actually one can prove $([1,(1969)],[2]$, and also Sect. 3 of the present paper where this argument is applied) the nonlinear stability, in such a form, of stationary solutions for which $C_{2} \geqq \Phi^{\prime \prime} \geqq C_{1}>0$.

In the presence of symmetry other first integrals are available. For instance in the case of a plane periodic flow between two parallel plates, the following functional

$$
J=\int_{D} y \omega d x d y
$$

is a first integral. It is natural to investigate how the above variational argument works for this functional.

\section{Defining}

$$
\hat{J}=J+\int \Phi(\omega) d x d y,
$$

the condition

$$
\delta \hat{J}(\bar{u})=0
$$

implies

$$
-\Phi^{\prime}(\bar{\omega})=y, \quad-\Phi^{\prime \prime}(\bar{\omega})=\left(\partial_{y} \bar{\omega}\right)^{-1}
$$

for $\bar{\omega}=\bar{\omega}(x, y)$ depending only on $y$.

We assume the condition

$$
C_{2} \geqq-\left(\partial_{y} \bar{\omega}\right)^{-1} \geqq C_{1}>0,
$$

and extend the function $\Phi$ out of the range of $\bar{\omega}$ to a smooth function, again denoted by $\Phi$, satisfying the condition $C_{1}^{-1} \geqq \Phi^{\prime \prime} \geqq C_{2}^{-1}$. Then the second variation

$$
\delta^{2} \hat{J}=-\int\left(\partial_{y} \bar{\omega}\right)^{-1}(\delta \omega)^{2} d x d y
$$

is positive. For a perturbation $\omega_{t}$ of the equilibrium, we have

$$
\begin{aligned}
\hat{J}\left(\omega_{t}\right)-\hat{J}(\bar{\omega})= & \int_{D} y(\omega-\bar{\omega})+\int_{D}[\Phi(\omega)-\Phi(\bar{\omega})] \\
= & \int_{D}\left[y(\omega-\bar{\omega})+\Phi^{\prime}(\bar{\omega})(\omega-\bar{\omega})\right] \\
& +\int_{D}\left[\Phi(\omega)-\Phi(\bar{\omega})-\Phi^{\prime}(\bar{\omega})(\omega-\bar{\omega})\right] .
\end{aligned}
$$


The first term on the right-hand side of $(1.11)$ is zero (because $\delta \hat{J}=0$ ). Furthermore

$$
\begin{aligned}
\left\|\omega_{1}-\bar{\omega}\right\|_{2}^{2} & \leqq 2 C_{2}\left(\hat{J}\left(\omega_{t}\right)-\hat{J}(\bar{\omega})\right) \quad \text { by }(1.11) \text { and Taylor's formula, } \\
& \leqq 2 C_{2}\left(\hat{J}\left(\omega_{0}\right)-\hat{J}(\bar{\omega})\right) \quad \text { by time invariance, } \\
& \leqq C_{2} C_{1}^{-1}\left\|\omega_{0}-\bar{\omega}\right\|_{2}^{2} \quad \text { again by (1.11) and Taylor's formula. }
\end{aligned}
$$

Therefore we have simply proved the nonlinear stability under assumption (1.10).

The above result can be inproved (considering $\hat{H}+\lambda J$ as Lyapunov function, $[1,1966])$ to obtain the stability under a weaker assumption.

As pointed out in [2], the above method works under smoothness assumptions on the equilibrium solution $\bar{\omega}$ and in case of bounded domains. Nevertheless, there are physically interesting situations (for instance cases in which the vorticity is the characteristic function of a strip and hence the velocity is piecewise linear) which cannot be studied by means of such a method. Generalization to nonsmooth cases do not seem immediate and are not available in the literature, except for the case of the so-called vortex patch, i.e. when $\bar{\omega}$ is the characteristic function of a disk [3] (see also [4-6]). In [3] two different methods to obtain the stability (in the $L_{1}$ norm) of a circular vortex patch are presented. One, based on the conservation of the angular momentum and its minimum properties with respect to isorotational perturbations, makes use of the symmetry of the problem and is very simple. The other, based on the energy conservation law, is much more intricate but in some sense gives much more information, as we shall see later.

In the present paper (Sect. 2) we give sufficient conditions for the nonlinear stability (in the $L_{1}$ sense) of any monotone, possibly nonsmooth profile of vorticity in symmetric domains. This fully exploits the ideas contained in the angular momentum method in [3].

In Sect. 3 we discuss the stability of smooth solutions in the presence of perturbations of the boundary, by means of the Arnold method. Moreover, we obtain the same result for vortex patches as a corollary of the energy method presented in [3]. In this case the (simpler) angular momentum method is useless because perturbations of the boundary destroy the symmetry.

\section{2.}

We consider an ideal incompressible fluid with unit density in the bounded domain $\Lambda \subset \mathbb{R}^{2}$ :

$$
\Lambda=\{(x, y) \mid 0<x<L, 0<y<A\} .
$$

The velocity field $u=\left(u^{(1)}, u^{(2)} \mid \in \mathbb{R}^{2}\right.$ can be described in terms of the stream function $\Psi$ for which $u=\nabla^{\perp} \Psi=\left(\partial_{y} \Psi,-\partial_{x} \Psi\right)$.

Denoting by $\omega=\partial_{x} u^{(2)}-\partial_{y} u^{(1)}$ the vorticity, we have

$$
-\Delta \Psi=\omega \text {. }
$$

We assume periodic boundary conditions in $x=0, x=L$ and impermeability conditions in $y=0, y=A$. Hence, denoting by $G_{A} \omega$ the stream function solution of Eq. (2.2), we have:

$$
\begin{gathered}
\left(G_{\Lambda} \omega\right)(0, y)=\left(G_{\Lambda} \omega\right)(L, y), \\
\left(G_{\Lambda} \omega\right)(x, 0)=0 \quad\left(G_{\Lambda} \omega\right)(x, A)=\alpha .
\end{gathered}
$$


We choose $\alpha=0$ adding a suitable potential flow.

The vorticity evolves according to the Euler equation,

$$
\left\{\begin{array}{l}
\partial_{t} \omega+(u \cdot \nabla) \omega=0 \\
u=\nabla^{\perp} G_{\Lambda} \omega .
\end{array}\right.
$$

It is well known that the initial value problem associated to Eq. (2.4) can be uniquely solved (in a weak sense) for initial conditions $\omega_{0} \in L_{\infty}$. Moreover, denoting by $\gamma_{t}(x, y)$ the solution of the ordinary differential equation $\dot{\gamma}_{t}(x, y)$ $=u_{t}\left(\gamma_{t}(x, y)\right), \gamma_{0}(x, y)=(x, y)$, we have $\omega_{t}(x, y)=\omega_{0}\left(\gamma_{-t}(x, y)\right)$, where $\omega_{t}$ solves Eq. (2.4) with initial datum $\omega_{0}$.

Due to the symmetry of the problem, the following quantity,

$$
J\left(\omega_{t}\right)=\int_{0}^{L} d x \int_{0}^{A} d y y \omega_{t}(x, y),
$$

is preserved during the motion. This can be verified by a direct computation. More generally, constant of motion which are momentum maps for the symmetry group in the Hamiltonian formalism, have been investigated in [7].

Moreover, any stationary solution $\bar{\omega}(x, y)=\bar{\xi}(y)$ which is non-increasing is also an absolute minimum for the functional $J$ in the class of all isorotational vorticity fields (see the definition below). These two are the basic ingredients for our stability results that can be formulated as follows.

Theorem 1. Let $\bar{\omega}(x, y)=\bar{\xi}(y)$ be nonincreasing, $\bar{\omega} \in L_{\infty}$. Then $\bar{\omega}$ is stable in the $L_{1}$ sense, with respect to $L_{\infty}$ perturbations. This means that, for all $\varepsilon>0$ there exists $\delta>0$ such that if

$$
\omega_{0} \in L_{\infty} \text { and }\left\|\bar{\omega}-\omega_{0}\right\|_{1}<\delta
$$

then

$$
\sup _{t \geqq 0}\left\|\bar{\omega}-\omega_{t}\right\|_{1}<\varepsilon .
$$

Remark. As it will follow by the proof, the same result holds for nondecreasing $\bar{\xi}$. Therefore, any monotone profile is stable in the above sense.

The proof of Theorem 1 is a straightforward consequence of the following two lemmas whose proof will be postponed to the end of the section.

We define the family of the isorotational profiles

$$
\begin{aligned}
\mathscr{J}(\bar{\omega}) & =\{\omega \mid \forall \lambda, \text { meas }\{(x, y) \mid \omega(x, y)>\lambda\} \\
& =\operatorname{meas}\{(x, y) \mid \bar{\omega}(x, y)>\lambda\}\} .
\end{aligned}
$$

Lemma 1. Suppose $\bar{\omega}$ is as in Theorem 1 and $\omega \in \mathscr{J}(\bar{\omega})$. Then

$$
\frac{1}{2 L \alpha}\|\omega-\bar{\omega}\|_{1}^{2} \leqq J(\omega)-J(\bar{\omega}) \leqq A\|\omega-\bar{\omega}\|_{1},
$$

where $\alpha=\|\bar{\omega}\|_{\infty}$.

Lemma 2. Suppose $\bar{\omega}$ is as in Theorem $1, \omega_{0} \in L_{\infty}$. Then there exists $\tilde{\omega}(x, y)=\tilde{\xi}(y)$ such that $\omega_{0} \in \mathscr{J}(\tilde{\omega})$ and if $\left\|\bar{\omega}-\omega_{0}\right\|_{1}<\delta$, then $\|\tilde{\omega}-\bar{\omega}\|_{1}<\delta$.

Now we are able to prove Theorem 1. 
Proof of Theorem 1. Putting $\left\|\omega_{0}\right\|_{\infty}=\left\|\omega_{t}\right\|_{\infty}=\alpha$ and taking into account, by virtue of the Liouville theorem, that $\omega_{t} \in \mathscr{J}(\tilde{\omega})$ if $\omega_{0} \in \mathscr{J}(\tilde{\omega})$, we have:

$$
\begin{aligned}
\left\|\omega_{t}-\bar{\omega}\right\|_{1} & \leqq\left\|\omega_{t}-\tilde{\omega}\right\|_{1}+\|\tilde{\omega}-\bar{\omega}\|_{1} \\
& \leqq \sqrt{2 \alpha L\left(J\left(\omega_{t}\right)-J(\tilde{\omega})\right)}+\left\|\omega_{0}-\bar{\omega}\right\|_{1}
\end{aligned}
$$

(by Lemmas 1 and 2)

$$
\leqq \sqrt{2 \alpha L\left(J\left(\omega_{0}\right)-J(\tilde{\omega})\right)}+\left\|\omega_{0}-\bar{\omega}\right\|_{1}
$$

(by the time invariance of $J$ )

$$
\leqq \sqrt{2 \alpha L A\left\|\omega_{0}-\tilde{\omega}\right\|_{1}}+\left\|\omega_{0}-\bar{\omega}\right\|_{1}
$$

(by Lemma 1)

$$
\leqq \sqrt{2 \alpha L A\left(\left\|\omega_{0}-\bar{\omega}\right\|_{1}+\|\bar{\omega}-\tilde{\omega}\|_{1}\right)}+\left\|\omega_{0}-\bar{\omega}\right\|_{1}
$$

(by Lemma 2)

$$
\leqq 2 \sqrt{\alpha L A\left\|\omega_{0}-\bar{\omega}\right\|_{1}}+\left\|\omega_{0}-\bar{\omega}\right\|_{1} \text {. }
$$

Proof of Lemma 1. We shall assume, without losing generality, $\bar{\xi}(A)=0$. Let us define

$$
\begin{aligned}
& \bar{A}_{k}=\left\{(x, y) \mid \bar{\omega}(x, y)>\frac{k \alpha}{N}, k=1 \ldots N-1\right\}, \\
& A_{k}=\left\{(x, y) \mid \omega(x, y)>\frac{k \alpha}{N}, k=1 \ldots N-1\right\}, \\
& \bar{\omega}^{N}=\sum_{k=1}^{N-1} \frac{\alpha}{N} X_{\bar{A}_{k}}, \quad \omega^{N}=\sum_{k=1}^{N-1} \frac{\alpha}{N} X_{A_{k}},
\end{aligned}
$$

where, as usual, $X_{B}$ denotes the characteristic function of the set $B$. Obviously $\bar{A}_{k}$ $\supset \bar{A}_{h}$ and $A_{k} \supset A_{h}$ if $k<h$. We have

$$
\begin{aligned}
J\left(\omega^{N}\right)-J\left(\bar{\omega}^{N}\right) & =\frac{\alpha}{N} \sum_{k=1}^{N-1}\left(\int_{A_{k}} y d x d y-\int_{\bar{A}_{k}} y d x d y\right) \\
& =\frac{\alpha}{N} \sum_{k=1}^{N-1}\left(\int_{A_{k} \backslash \bar{A}_{k}} y d x d y-\int_{\bar{A}_{k} \backslash A_{k}} y d x d y\right) .
\end{aligned}
$$

Defining $y_{k}$ in such a way that

$$
\bar{A}_{k}=\left\{(x, y) \mid 0 \leqq x \leqq L, 0 \leqq y<y_{k}\right\},
$$

and realizing that, by virtue of the isorotationality,

$$
\operatorname{meas}\left(A_{k} \backslash \bar{A}_{k}\right)=\operatorname{meas}\left(\bar{A}_{k} \backslash A_{k}\right) \equiv \beta_{k},
$$

we have

$$
\begin{aligned}
J\left(\omega^{N}\right)-J\left(\bar{\omega}^{N}\right) & \geqq \frac{L \alpha}{N} \sum_{k=1}^{N-1}\left(\int_{y_{k}}^{y_{k}+\frac{\beta_{k}}{L}}-\int_{y_{k}-\frac{\beta_{k}}{L}}^{y_{k}} y d y\right) \\
& =\frac{L \alpha}{N} \sum_{k=1}^{N-1} \beta_{k}^{2} L^{-2} \\
& =\frac{L^{-1} \alpha}{2 N} \sum_{k=1}^{N-1}\left\|X_{A_{k}}-X_{\bar{A}_{k}}\right\|_{1}^{2} .
\end{aligned}
$$


On the other side, by the Cauchy-Schwartz inequality,

$$
\begin{aligned}
\left\|\bar{\omega}^{N}-\omega^{N}\right\|_{1} & =\int d x d y\left|\sum_{k=1}^{N-1} \frac{\alpha}{N}\left(X_{A_{k}}-X_{\bar{A}_{k}}\right)\right| \\
& \leqq \sum_{k=1}^{N-1} \frac{\alpha}{N}\left\|X_{A_{k}}-X_{\bar{A}_{k}}\right\|_{1} \\
& \leqq \frac{\alpha}{N} \sqrt{N-1} \sqrt{\sum_{k}\left\|X_{A_{k}}-X_{\bar{A}_{k}}\right\|_{1}^{2}},
\end{aligned}
$$

and hence

$$
J\left(\omega^{N}\right)-J\left(\bar{\omega}^{N}\right) \geqq \frac{1}{2 L \alpha}\left\|\bar{\omega}^{N}-\omega^{N}\right\|_{1}^{2} .
$$

The left-hand side of inequality (2.9) follows by passing to the limit $N \rightarrow \infty$, by the Dominated Convergence Theorem. The right-hand side of inequality (2.9) is trivial.

Proof of Lemma 2. We construct $\tilde{\omega}$ in the following way. Defining

$$
\begin{aligned}
A_{\lambda} & =\left\{(x, y) \mid \omega_{0}(x, y)>\lambda\right\}, \\
y(\lambda) & =\frac{\operatorname{meas} A_{\lambda}}{L} \in[0, A], \\
\tilde{\xi}(y) & =\sup \left\{\lambda \mid y(\lambda) \geqq y, \lambda \in\left(\operatorname{essinf} \omega_{0}, \operatorname{ess} \sup \omega_{0}\right),\right. \\
\tilde{\omega}(x, y) & =\widetilde{\xi}(y),
\end{aligned}
$$

we realize that $\tilde{\xi}$ is nonincreasing and $\omega_{0} \in \mathscr{J}(\tilde{\omega})$. We define

$$
\begin{aligned}
S(\lambda, \omega) & =\{(x, y) \mid \omega(x, y)>\lambda\}, \\
\sigma(\lambda) & =\operatorname{meas}(S(\lambda, \tilde{\omega}) \Delta S(\lambda, \bar{\omega})), \\
\Sigma(\lambda) & =\operatorname{meas}\left(S(\lambda, \bar{\omega}) \Delta S\left(\lambda, \omega_{0}\right)\right),
\end{aligned}
$$

and observe that, because of the monotonicity of $\bar{\omega}$ and $\tilde{\omega}, S(\lambda, \tilde{\omega})$ and $S(\lambda, \bar{\omega})$ are rectangles, one of which subset of the other. Therefore,

$$
\begin{aligned}
\sigma(\lambda) & =\int\left|X_{S(\lambda, \tilde{\omega})}-X_{S(\lambda, \tilde{\omega})}\right| d x d y \\
& =\left|\int\left(X_{S(\lambda, \tilde{\omega})}-X_{S(\lambda, \tilde{\omega})}\right) d x d y\right|=\left|\int\left(X_{S\left(\lambda, \omega_{0}\right)}-X_{S(\lambda, \tilde{\omega})}\right)\right| \\
& \leqq \int\left|X_{S\left(\lambda, \omega_{0}\right)}-X_{S(\lambda, \bar{\omega})}\right| \leqq \Sigma(\lambda) .
\end{aligned}
$$

Finally

$$
\|\tilde{\omega}-\bar{\omega}\|_{1}=\int d \lambda \sigma(\lambda) \leqq \int d \lambda \Sigma(\lambda)=\left\|\bar{\omega}-\omega_{0}\right\|_{1} .
$$

We conclude this section by remarking that the above technique can be applied to the case of circularly symmetric distribution of vorticity in circularly symmetric domains and, probably, to other cases when symmetries are present.

Let us consider the region

$$
\Lambda=\left\{z \in \mathbb{R}^{2}|r<| z \mid<R\right\}, \quad+\infty>R>0, \quad r \geqq 0 .
$$


Denoting by $\varrho, \theta$ the polar coordinates, consider $\bar{\omega}(\varrho, \theta)=\bar{\xi}(\varrho)$ a circularly symmetric stationary solution. The angular momentum defined as

$$
J_{\theta}\left(\omega_{t}\right)=\int \varrho^{3} \omega_{t}(\varrho, \theta) d \varrho d \theta
$$

is a constant of motion. Furthermore, given $\bar{\xi}$ nonincreasing in $\varrho$, the function $J$, restricted on $J(\bar{\omega})$ takes in $\bar{\omega}$ its minimum value.

The following theorem generalizes the result in [3]. The proof is a straightforward copy of the proof of Theorem 1.

Theorem 2. Any stationary $\bar{\omega}(\varrho, \theta)=\bar{\xi}(\varrho)$, essentially bounded and nonincreasing (nondecreasing), is stable in the $L_{1}$ sense with respect to $L_{\infty}$ perturbations.

Remark. If $R=+\infty$, the same result can be obtained either by considering perturbations of compact support, or (what is essentially the same) by weighting suitably the $L_{1}$ norm [4].

\section{3.}

In this section we investigate the stability of a stationary Euler flow with respect to perturbations including small deformations of the domain containing the fluid. To do this, we modify suitably the Arnold method and make use of continuity properties of the Green function for the Dirichlet problem. In this way we find sufficient conditions which ensure the stability of smooth stationary flows also under perturbations of the boundary. The non-smooth symmetric cases discussed in the previous section cannot be treated simply, because perturbations of the boundary destroy the symmetry. This makes difficult the use of linear or angular momentum as Lyapunov functions, since they are no longer conserved quantities. Nevertheless, the only case in which the energy method is available for a nonsmooth situation, that is the circular vortex patch [3], can be treated also allowing perturbations of the boundary. This remark shows how the energy method is more powerful although, technically speaking, more difficult to deal with.

We begin by discussing the smooth case.

Let $u, \omega=\operatorname{curl} u$, be a smooth solution of the planar Euler equation in a bounded, open domain $D$ with external boundary $\mathscr{C}_{0}$ encircling holes $D_{i}$ with smooth boundaries $\mathscr{C}_{i}$. The boundary conditions are, as usual, $\left.u \cdot n\right|_{\mathscr{C}_{i}}=0(n$ is the inner normal). The stream function $\Psi$, such that $u=\nabla^{\perp} \Psi$, is a well defined, single valued function, satisfying $\Delta \Psi=-\omega,\left.\Psi\right|_{\mathscr{C}_{0}}=0,\left.\Psi\right|_{\mathscr{C}_{i}}=\beta_{i}, i>0$, where $\beta_{i}$ are given by the circulations $\Gamma_{i}=\int_{\mathscr{C}_{i}} u \cdot d \ell$ (see [8, Theorem 6.1]). We consider now a stationary solution $\bar{u}, \bar{\omega}=\operatorname{curl} \bar{u}$, in a domain $\bar{D}$ and a time-dependent solution $u_{t}$, $\omega_{t}$ living in a slightly different domain $D$ (with the same topological structure as $D$ ). The following functional, for $\Phi$ and $a_{i}$ to be specified later,

$$
\hat{H}=\frac{1}{2} \int_{D} u^{2}-\frac{1}{2} \int_{\bar{D}} \bar{u}^{2}+\int_{D} \Phi(\omega)-\int_{\bar{D}} \Phi(\bar{\omega})+\sum_{i=0}^{n} a_{i}\left(\Gamma_{i}-\bar{\Gamma}_{i}\right),
$$

where

$$
\Gamma_{i}=\int_{\mathscr{C}_{2}} u \cdot d \ell, \quad \bar{\Gamma}_{i}=\int_{\overline{\mathscr{C}}_{2}} \bar{u} \cdot d \ell, \quad i=0 \ldots n
$$


is constant in time. We denote by the same symbols $u, \bar{u}, \omega, \bar{\omega}$ the fields extending $u$, $\bar{u}, \omega, \bar{\omega}$ as zero outside their domains of definition $D$ and $\bar{D}$. Making use of the identity $u^{2}-\bar{u}^{2}=(u-\bar{u})^{2}+2 \bar{u} \cdot(u-\bar{u})$, and defining $\tilde{\omega}=\omega X_{\bar{D}}$, we write

$$
\hat{H}=\sum_{i=1}^{5} F_{i}
$$

where

$$
\begin{aligned}
& F_{1}=\int_{\bar{D}} \bar{u} \cdot(\tilde{u}-\bar{u})+\int_{\bar{D}} \Phi^{\prime}(\bar{\omega})(\tilde{\omega}-\bar{\omega})+\sum_{i=0}^{n} a_{i}\left(\Gamma_{i}-\overline{\Gamma_{i}}\right), \\
& F_{2}=\int_{\bar{D}} \bar{u} \cdot(u-\tilde{u}), \\
& F_{3}=\int_{\mathbb{R}^{2}} \frac{\left(u X_{D}-\bar{u} X_{\bar{D}}\right)^{2}}{2}, \\
& F_{4}=\int_{\bar{D} \cap D}\left\{\Phi(\omega)-\Phi(\bar{\omega})-\Phi^{\prime}(\bar{\omega})\left(\omega X_{\bar{D}}-\bar{\omega}\right)\right\}, \\
& F_{5}=\int_{D \backslash \bar{D}} \Phi(\omega)-\int_{\bar{D} \backslash D} \Phi(\bar{\omega})-\int_{\bar{D} \backslash D} \Phi^{\prime}(\bar{\omega})(\tilde{\omega}-\bar{\omega}) .
\end{aligned}
$$

Here $\tilde{u}$ denotes a vector field defined in $\bar{D}$, tangent on $\partial \bar{D}$, having vorticity $\tilde{\omega}$ and circulations $\tilde{\Gamma_{i}} \equiv \int_{\tilde{\mathscr{C}}_{i}} \tilde{u} \cdot d \ell=\int_{\mathscr{C}_{i}} u \cdot d \ell \equiv \Gamma_{i}$. The conditions for $\Phi$ and $a_{i}$ are chosen so that $F_{1}=0$. One has:

$$
\begin{aligned}
& \int_{\bar{D}} \Phi^{\prime}(\bar{\omega})(\tilde{\omega}-\bar{\omega})= \int_{\bar{D}} \operatorname{curl}\left[\Phi^{\prime}(\bar{\omega})(\tilde{u}-\bar{u})\right] \\
&-\int_{\bar{D}} \Phi^{\prime \prime}(\bar{\omega}) \nabla^{\perp} \bar{\omega} \cdot(\tilde{u}-\bar{u}), \\
&\left.\sum_{i=0}^{k} \Phi^{\prime}(\bar{\omega})\right|_{\overline{\mathscr{C}}_{i}}\left(\tilde{\Gamma}_{i}-\overline{\Gamma_{i}}\right)-\int_{\bar{D}} \bar{u} \cdot(\tilde{u}-\bar{u})=0
\end{aligned}
$$

where we have chosen $\Phi$ (up to some constant value) in such a way that $\Phi^{\prime \prime}(\bar{\omega})=\frac{\bar{u}}{\nabla^{\perp} \bar{\omega}}$ (at the equilibrium $\bar{u}$ and $\nabla^{\perp} \bar{\omega}$ are collinear and such a choice is possible) and we have used the fact that $\bar{\omega}$ is constant on each $\partial \bar{D}_{i}$ because of the stationarity. Therefore, $F_{1}=0$ by an appropriate choice of the $a_{i}$ 's, being $\tilde{\Gamma}_{i}=\Gamma_{i}$.

Suppose the following condition holds:

$$
0<C_{1} \leqq \frac{\bar{u}}{\nabla^{\perp} \bar{\omega}} \equiv \Phi^{\prime \prime}(\bar{\omega}) \leqq C_{2}<+\infty .
$$

We extend $\Phi$, outside the range of $\bar{\omega}$, to a smooth function still denoted by $\Phi$ and satisfying (3.10). Under such hypothesis, our stability result follows by combining known arguments with uniform in time estimates on all the $F_{i}$ 's, which are formally vanishing when $D \rightarrow \bar{D}$.

We define

$$
d(D, \bar{D})=\max _{i} \max _{z \in \mathscr{C}_{i}} \min _{z^{\prime} \in \overline{\mathscr{C}}_{i}}\left|z-z^{\prime}\right|,
$$


and denote by $\eta_{i}, i=1,2, \ldots$ any positive constant (independent of $t$ ) depending only on $\omega_{0}, \bar{\omega}, d(D, \bar{D})$, the geometry and such that $\eta_{i} \rightarrow 0$ as $d(D, \bar{D}) \rightarrow 0$. Then:

$$
\begin{aligned}
\left|F_{5}\right| \leqq & \|\Phi\|_{\infty}(\operatorname{meas}(D \backslash \bar{D})+\operatorname{meas}(\bar{D} \backslash D)) \\
& +\left\|\Phi^{\prime}\right\|_{\infty}\left(\left\|\omega_{0}\right\|_{\infty}+\|\bar{\omega}\|_{\infty}\right) \\
& \cdot \operatorname{meas}(\bar{D} \backslash D) \leqq \eta_{1} .
\end{aligned}
$$

Let $Q \subset D \cap \bar{D}$ be a domain with $C^{2}$ boundary, such that meas $(\bar{D} \backslash Q) \leqq 2$ meas $(\bar{D} \backslash D)$ and the length of $\partial Q \leqq 2$ length of $\partial \bar{D}$. Then

$$
\left|F_{2}\right| \leqq \text { const }\|\bar{\omega}\|_{\infty}\left\|\omega_{0}\right\|_{\infty} \operatorname{meas}(\bar{D} \backslash D)+\left|\int_{Q} \bar{u}_{t} \cdot\left(u_{t}-\tilde{u}_{t}\right)\right| ;
$$

(having used the inequality $\|u\|_{\infty} \leqq \operatorname{const}\|\operatorname{curl} u\|_{\infty}$ ) therefore:

$$
\begin{aligned}
\left|F_{2}\right| \leqq \eta_{2} & +\left|\int_{\partial Q} \tilde{u}_{t}\left(G_{D} \omega_{t}-G_{\bar{D}} \omega_{t}\right)\right| \\
& +\left|\int_{Q} \bar{\omega}_{t}\left(G_{D} \omega_{t}-G_{\bar{D}} \omega_{t}\right)\right| .
\end{aligned}
$$

Here $G_{D}$ denotes the operator whose kernel is the fundamental solution of the Poisson equation with Dirichlet boundary conditions in $D$. Putting $G_{0}=G_{\mathbb{R}^{2}}$, $\gamma_{D}=G_{D}-G_{0}$, we have, for $z \in \partial Q$,

$$
\begin{aligned}
\left|\left(G_{D}-G_{\bar{D}}\right) \omega_{t}(z)\right|= & \left|\left(\gamma_{D}-\gamma_{\bar{D}}\right) \omega_{t}(z)\right|=\left|\gamma_{D} \omega_{t}(z)-\gamma_{D} \omega_{t}(\zeta)\right| \\
& +\left|G_{0}\left(\omega_{t} X_{D}\right)(\zeta)-G_{0}\left(\omega_{t} X_{\bar{D}}\right)\left(z^{\prime}\right)\right| \\
& +\left|\gamma_{\bar{D}} \omega_{t}(z)-\gamma_{\bar{D}} \omega_{t}\left(z^{\prime}\right)\right| \\
\leqq & \operatorname{const}\left(|\zeta-z|+\left|\zeta-z^{\prime}\right|+\left|z^{\prime}-z\right|\right)+\eta_{3}
\end{aligned}
$$

(where $\zeta \in \partial_{D}$ and $z^{\prime} \in \partial \bar{D}$, and the above constant depends only on $\left\|\omega_{0}\right\|_{\infty}$ ). Minimizing on $\zeta$ and $z^{\prime}$, we bound the right-hand side of (3.14) in terms of $\eta_{4}$. By the maximum principle

$$
\begin{gathered}
\left|F_{2}(t)\right| \leqq \eta_{2}+\text { const } \eta_{4}\left\|\omega_{0}\right\| \text { (length of } \bar{D} \text { ) } \\
+\eta_{4}\left\|\omega_{0}\right\| \text { meas } Q \leqq \eta_{5} .
\end{gathered}
$$

Now we are in position to prove the following Theorem.

Theorem 3. Suppose that condition (3.10) holds. Then, for any $\varepsilon>0$, there exists a $\delta>0$ such that

$$
\sup _{t \geqq 0}\left(\int_{\mathbb{R}^{2}}\left(u_{t} X_{D}-\bar{u} X_{\bar{D}}\right)^{2}+\int_{\mathbb{R}^{2}}\left(\omega_{t} X_{D}-\bar{\omega} X_{\bar{D}}\right)^{2}\right)<\varepsilon,
$$

provided that

$$
\max \left(d(D, \bar{D}), \int_{\mathbb{R}^{2}}\left(u_{0} X_{D}-\bar{u} X_{\bar{D}}\right)^{2}+\left(\omega_{0} X_{D}-\bar{\omega} X_{\bar{D}}\right)^{2}\right)<\delta .
$$


Proof.

$$
\begin{aligned}
\frac{1}{2} \int_{\mathbb{R}^{2}}\left(u_{t} X_{D}-\bar{u} X_{\bar{D}}\right)^{2} & +\int_{\mathbb{R}^{2}}\left(\omega_{t} X_{D}-\bar{\omega} X_{\bar{D}}\right)^{2} \\
\leqq & F_{3}(t)+2 C_{1}^{-1} F_{4}+\int_{D \Delta \bar{D}}\left(\omega_{t} X_{D}-\bar{\omega} X_{\bar{D}}\right)^{2} \\
\leqq & C_{3} \hat{H}(t)+C_{3}\left\{\left|F_{2}(t)\right|+\left|F_{5}(t)\right|\right\} \\
& \left.+\left(\left\|\omega_{0}\right\|_{\infty}^{2}+\|\bar{\omega}\|_{\infty}^{2}\right) \operatorname{meas}(D \Delta \bar{D})\right) \\
\leqq & C_{3} \hat{H}(0)+\eta_{6} \\
\leqq & C_{3}\left\{F_{2}(0)+F_{3}(0)+F_{4}(0)+F_{5}(0)\right\}+\eta_{6} \\
\leqq & C_{4}\left[F_{3}(0)+\int_{\bar{D} \Delta D}\left(\omega_{0}-\bar{\omega}\right)^{2}\right]+\eta_{7} \cdot \square
\end{aligned}
$$

We discuss now the stability of the circular vortex patch,

$$
\bar{\omega}(z)=\bar{M} X_{C}(z), \quad \bar{M}>0,
$$

where $C$ is a disk of radius $\gamma$, in the domain

$$
\bar{\Lambda}=\left\{z \in \mathbb{R}^{2}|| z \mid \leqq \bar{R}\right\} .
$$

Let $\omega_{t}$ be a solution of the Euler equation with initial value $\omega_{0}$, where $\omega_{0}$ is a vortex patch of the form

$$
\omega_{0}(z)=M X_{A}(z),
$$

and $A$ is a measurable set contained in $\mathbb{R}^{2}$. Such evolution takes place in the domain $\Lambda$, where $\Lambda$ is a slight perturbation of $\bar{\Lambda} . \partial \Lambda$ is assumed to be $C^{2}$ and

$$
\min _{z \in \partial \Lambda}|z|>R-\delta_{1}, \quad \max _{z \in \partial \Lambda}|z|<R+\delta_{1} .
$$

We consider also

$$
\Lambda_{1}=\left\{z \in \mathbb{R}^{2}|| z \mid \leqq R\right\},
$$

the minimal disk encircling $\partial \Lambda \cup \partial \bar{\Lambda}$. Then, as $\delta_{1} \rightarrow 0, \mathrm{R} \rightarrow \bar{R}$.

Theorem 4. For any $\varepsilon>0$, there exists $\delta>0$ such that

$$
\sup _{t \geqq 0}\left\|\omega_{t}-\bar{\omega}\right\|_{1}<\varepsilon
$$

provided that

$$
\max \left(\left\|\omega_{0}-\bar{\omega}\right\|_{1}, \delta_{1}\right)<\delta .
$$

Proof. The proof is based on the (static) inequality (see [3]):

$$
C\|\omega-\bar{\omega}\|_{1}^{2} \leqq E_{D}(\bar{\omega})-E_{D}(\omega) \leqq C_{2}
$$

for $\|\omega-\bar{\omega}\|_{1}<a$, a sufficiently small. Here $D$ is any circular domain containing the support of $\omega$ and $\bar{\omega}, \bar{\omega}$ is a circular vortex patch, $\omega=\bar{M} X_{B}$ is a vortex patch, $B \subset D$, meas $B=$ meas $C, E_{D}$ is the energy functional, defined as $E_{D}(\omega)$ $=\frac{1}{2} \int \omega(z)\left(G_{D} \omega\right)(z) d z$. Let $\tilde{\omega}$ be a circular vortex patch such that $\|\tilde{\omega}\|_{\infty}=\left\|\omega_{t}\right\|_{\infty}$ and $\|\tilde{\omega}\|_{1}=\left\|\omega_{t}\right\|_{1}$. Since $\tilde{\omega}$ and $\bar{\omega}$ are both disks, it is:

$$
\|\tilde{\omega}-\bar{\omega}\|_{1} \leqq\left\|\omega_{0}-\bar{\omega} \cdot\right\|_{1} .
$$


Then, we have, supposing $t$ and $\delta$ sufficiently small such that $\left\|\omega_{t}-\tilde{\omega}\right\|_{1}<a$,

$$
\begin{aligned}
\left\|\omega_{t}-\bar{\omega}\right\|_{1} & \leqq\left\|\tilde{\omega}-\omega_{t}\right\|_{1}+\|\tilde{\omega}-\bar{\omega}\|_{1} \\
& \leqq \frac{1}{\sqrt{C}} \sqrt{E_{\Lambda_{1}}(\tilde{\omega})-E_{\Lambda_{1}}\left(\omega_{t}\right)}+\left\|\omega_{0}-\bar{\omega}\right\|_{1} \quad[\operatorname{by~}(3.26),(3.27)] \\
& \leqq \frac{1}{\sqrt{C}}\left\{\sqrt{\left|E_{\Lambda_{1}}(\tilde{\omega})-E_{\Lambda}(\tilde{\omega})\right|}+\sqrt{\left|E_{\Lambda}(\tilde{\omega})-E_{\Lambda}\left(\omega_{t}\right)\right|}\right. \\
& \left.+\sqrt{\left|E_{\Lambda}\left(\omega_{t}\right)-E_{\Lambda_{1}}\left(\omega_{t}\right)\right|}\right\}+\left\|\omega_{0}-\bar{\omega}\right\|_{1} .
\end{aligned}
$$

By the same arguments leading to (3.14) and (3.15) and by the use of the conservation in time of the $L_{\infty}$ norm, we can get a function $\alpha$ depending only on $\omega_{0}$, $\delta_{1}$, and $\bar{R}$ such that

$$
\begin{gathered}
\sqrt{\left|E_{\Lambda_{1}}(\tilde{\omega})-E_{\Lambda}(\tilde{\omega})\right|}+\sqrt{\left|E_{\Lambda}\left(\omega_{t}\right)-E_{\Lambda_{1}}\left(\omega_{t}\right)\right|} \leqq \alpha, \\
\lim _{\delta_{1} \rightarrow 0} \alpha=0 .
\end{gathered}
$$

Moreover, by the time invariance of $E_{A}$ :

$$
\begin{aligned}
\left|E_{\Lambda}(\tilde{\omega})-E_{\Lambda}\left(\omega_{t}\right)\right|= & \left|E_{\Lambda}(\tilde{\omega})-E_{\Lambda}\left(\omega_{0}\right)\right| \\
= & \frac{1}{2} \mid \int d z d z^{\prime} \tilde{\omega}(z) g_{\Lambda}\left(z, z^{\prime}\right) \tilde{\omega}\left(z^{\prime}\right) \\
& -\int d z d z^{\prime} \omega_{0}(z) g_{\Lambda}\left(z, z^{\prime}\right) \omega_{0}\left(z^{\prime}\right) \mid \\
\leqq & \frac{1}{2}\left\|\omega_{0}-\tilde{\omega}\right\|_{1}\left\{\sup _{z} \int\left|g_{\Lambda}\left(z, z^{\prime}\right) \tilde{\omega}\left(z^{\prime}\right)\right| d z^{\prime}\right. \\
& \left.+\sup _{z} \int\left|g_{\Lambda}\left(z, z^{\prime}\right) \omega_{0}\left(z^{\prime}\right)\right| d z^{\prime}\right\} \\
\leqq & C_{1}\left(\|\tilde{\omega}\|_{\infty}+\left\|\omega_{0}\right\|_{\infty}\right)\left\|\omega_{0}-\tilde{\omega}\right\|_{1}
\end{aligned}
$$

for some $C_{1}>0$, where $\left(G_{\Lambda} \omega\right)(z)=\int g_{\Lambda}\left(z, z^{\prime}\right) \omega\left(z^{\prime}\right) d z^{\prime}$. Therefore, by (3.30) and (3.31)

$$
\begin{aligned}
\left\|\omega_{t}-\bar{\omega}\right\|_{1} \leqq & \left\|\omega_{0}-\bar{\omega}\right\|_{1}+\frac{\alpha}{\sqrt{C}} \\
& +\frac{\sqrt{2 C_{1}\left\|\omega_{0}\right\|_{\infty}}}{\sqrt{C}} \sqrt{\left\|\omega_{0}-\tilde{\omega}\right\|_{1}} \\
\leqq & \left\|\omega_{0}-\bar{\omega}\right\|_{1}+\frac{\alpha}{\sqrt{C}} \\
& +2 \sqrt{\frac{C_{1}\left\|\omega_{0}\right\|_{\infty}}{C}} \sqrt{\left\|\omega_{0}-\bar{\omega}\right\|_{1}} .
\end{aligned}
$$

But

$$
\begin{aligned}
\left\|\omega_{t}-\tilde{\omega}\right\|_{1} & \leqq\left\|\omega_{t}-\bar{\omega}\right\|_{1}+\|\tilde{\omega}-\tilde{\omega}\|_{1} \\
& \leqq\left\|\omega_{t}-\bar{\omega}\right\|_{1}+\left\|\bar{\omega}-\omega_{0}\right\|_{1},
\end{aligned}
$$

thus by (3.32) $\left\|\omega_{t}-\tilde{\omega}\right\|_{1} \leqq a$ for all times and this achieves the proof. 
Acknowledgements. One of us (C.M.) is grateful to Prof. N. Kuiper for the kind hospitality at the IHES, where part of this research has been done.

\section{References}

1. Arnold, V.: Dokl. Nat. Nauk. 162, 773 (1965); J. Mec. 5, 29 (1966); Am. Math. Soc. Transl. 79, 267 (1969)

2. Holm, D.D., Marsden, J.E., Ratiu, T., Weinstein, A.: Nonlinear stability of fluid and plasma systems. Phys. Rep. (1985, in press)

3. Wan, Y.H., Pulvirenti, M.: Nonlinear stability of circular vortex patches. Commun. Math. Phys. 99, 435-450 (1985)

4. Wan, Y.H.: Contemp. Math. 28 (1984)

5. Benfatto, G., Marchioro, C., Pulvirenti, M.: Vortex methods in planar fluid dynamics. Proc. of "Applications of Mathematics in Technology" Roma. Stuttgart: G.B. Teubner 1984

6. Tang, Y.: Nonlinear stability of vortex patches. Thesis, SUNY, Buffalo (1984)

7. Mardsen, J.E., Weinstein, A.: Coadjoint orbits, vortices, and Clebsch variables for incompressible fluids. Physica 7D, 305 (1983)

8. Meyer, E.R.: Introduction to mathematical fluid dynamics. New York: Wiley 1971

Communicated by J. L. Lebowitz

Received December 5, 1984 